\title{
LIFTING CHERN CLASSES BY MEANS OF EKEDAHL-OORT STRATA
}

\author{
GERARD VAN DER GEER AND EDUARD LOOIJENGA
}

\begin{abstract}
The moduli space $\mathcal{A}_{g}$ of principally polarized abelian varieties of genus $g$ is defined over $\mathbb{Z}$ and admits a minimal compactification $\mathcal{A}_{g}^{*}$, also defined over $\mathbb{Z}$. The Hodge bundle over $\mathcal{A}_{g}$ has its Chern classes in the Chow ring of $\mathcal{A}_{g}$ with $\mathbb{Q}$-coefficients. We show that over $\mathbb{F}_{p}$, these Chern classes naturally lift to $\mathcal{A}_{g}^{*}$ and do so in the best possible way: despite the highly singular nature of $\mathcal{A}_{g}^{*}$ they are represented by algebraic cycles on $\mathcal{A}_{g}^{*} \otimes \mathbb{F}_{p}$ which define elements in the bivariant Chow ring. This is in contrast to the situation in the analytic topology, where these Chern classes have canonical lifts to complex cohomology of the minimal compactification as Goresky-Pardon classes, which are known to define nontrivial Tate extensions inside the mixed Hodge structure on this cohomology.
\end{abstract}

\section{InTRODUCTION AND STATEMENT OF THE MAIN RESUlT}

Few objects in algebraic geometry have such a rich structure as the moduli space $\mathcal{A}_{g}$ of principally polarized abelian varieties of dimension $g$. Its modular interpretation makes it a stack over $\mathbb{Z}$ and it comes as such with a rank $g$ vector bundle, the Hodge bundle $\mathbb{E}^{g}$ (which we may regard as the basic automorphic bundle over $\mathcal{A}_{g}$ in the sense that all other such over $\mathcal{A}_{g}$ are manufactured from it). Its determinant bundle $\operatorname{det}\left(\mathbb{E}^{g}\right)$ is ample and when $g \geq 2$, the graded algebra of automorphic forms $\oplus_{N=0}^{\infty} H^{0}\left(\mathcal{A}_{g},\left(\operatorname{det}\left(\mathbb{E}^{g}\right)^{\otimes N}\right)\right.$ is finitely generated so that its Proj defines a natural projective completion $\mathcal{A}_{g}^{*}$ of $\mathcal{A}_{g}$. The complex-analytic space $\mathcal{A}_{g}^{\text {an }}$ underlying $\mathcal{A}_{g} \otimes \mathbb{C}$ has the familiar description as the quotient of the Siegel upper half space $D_{g}$ of genus $g$ by the integral symplectic group $\operatorname{Sp}_{2 g}(\mathbb{Z})$ and $\mathcal{A}_{g}^{* \text { an }}$ is then the Satake-Baily-Borel compactification. Since $\mathcal{A}_{g}$ is a Deligne-Mumford stack, the (operational) Chow ring of $\mathcal{A}_{g}$ with $\mathbb{Q}$-coefficients, $A_{\mathbb{Q}}^{\bullet}\left(\mathcal{A}_{g}\right)$ is well-defined. The Chern classes of $\mathbb{E}^{g}$ generate a subalgebra $\mathcal{R}_{g}$ herein (we recall its presentation below). Since every automorphic bundle over $\mathcal{A}_{g}$ is universally expressed via a Schur functor in terms of its Hodge bundle, $\mathcal{R}_{g}$ contains the Chern classes of all such bundles. This is why we refer to $\mathcal{R}_{g}$ as the tautological ring of $\mathcal{A}_{g}$.

1991 Mathematics Subject Classification. Primary 11G18, 14 G35.

Key words and phrases. Chern classes, Baily-Borel compactification, Ekedahl-Oort strata.

E.L. was supported by the Chinese National Science Foundation. 
The cycle map embeds this ring in $H^{\bullet}\left(\mathcal{A}_{g}^{\text {an }} ; \mathbb{Q}\right)$ and its image can be characterized in several ways. One is that after tensoring with $\mathbb{R}$, it is the subalgebra representable by differential forms whose pull-backs to $D_{g}$ are $\mathrm{Sp}_{2 g}(\mathbb{R})$-invariant (such forms are automatically closed). Charney and Lee [3] had shown in 1983 that in the stable range (that is, for cohomological degree $<g$ ) these classes are liftable to $H^{\bullet}\left(\mathcal{A}_{g}^{* \text { an }} ; \mathbb{Q}\right)$, but Goresky and Pardon [13] proved in 2002 that they admit in fact a natural lift, provided we use the complex cohomology of $\mathcal{A}_{g}^{* a n}$. They raised the question whether their lifts lie in the rational cohomology. The answer to that question, given by one of us [17], is in general no. To see why, it is better to use the Chern characters rather than the Chern classes, for then the even indexed Chern characters are zero, so that the issue regarding liftability only concerns the odd indexed ones. The answer is then that for $k=2 r+1$ odd, the Goresky-Pardon lift $\operatorname{ch}_{k}^{\mathrm{gp}}\left(\mathbb{E}^{g}\right)$ of $\operatorname{ch}_{k}\left(\mathbb{E}^{g}\right)$ lies in the Hodge space $F^{k} H^{2 k}\left(\mathcal{A}_{g}^{* \text { an }}\right) \subset H^{2 k}\left(\mathcal{A}_{g}^{* \text { an }} ; \mathbb{C}\right)$. If we are also in the stable range $0<k<g / 2$, then, as we recall below, it lies in fact in the complexification of a mixed Tate substructure of $H^{2 k}\left(\mathcal{A}_{g}^{* \text { an }}\right)$ : an extension of $\mathbb{Q}(-k)$ by $\mathbb{Q}(0)$. This extension is nontrivial in the sense that it is proportional to a standard nontrivial one whose invariant is given by $(2 \pi \sqrt{-1})^{-k} \pi^{-k} \zeta(k)$. Since $k$ is odd, this implies that in this range, $\operatorname{ch}_{k}^{\mathrm{gp}}\left(\mathbb{E}^{g}\right)$ will not even be a real cohomology class.

We noted already back in 2015 that the situation is entirely different for $\mathcal{A}_{g} \otimes \mathbb{F}_{p}$. For this, let us recall that Ekedahl and van der Geer [4] had proved that $\mathcal{R}_{g}$ is then generated by the Ekedahl-Oort strata. Our observation at the time was that these strata intersect the boundary of $\mathcal{A}_{g}^{*} \otimes \mathbb{F}_{p}$ transversally with respect to its natural stratification (with "minimal perversity"), which means that these classes naturally lift to $\ell$-adic cohomology classes on $\mathcal{A}_{g}^{*} \otimes$ $\mathbb{F}_{p}$. We then realized that the notion of an $F$-zip, introduced by Moonen and Wedhorn in [18] and the classifying space of such as introduced by PinkWedhorn-Ziegler [21] make it fit into an even neater picture. This classifying space of zips is an Artin stack, denoted $\left[E_{\mathcal{Z}} \backslash \mathrm{Sp}_{2 g} \otimes \mathbb{F}_{p}\right]$ (we give more details below), which can be regarded as the characteristic $p$ counterpart of the compact dual $\check{D}_{g}$ of the Siegel upper space $D_{g}$. The Chow ring $\check{\mathcal{R}}_{g}:=$ $A_{\mathbb{Q}}^{\bullet}\left(\left[E_{\mathcal{Z}} \backslash \mathrm{Sp}_{2 g} \otimes \mathbb{F}_{p}\right]\right)$ is isomorphic to the one of $\check{D}_{g}$. We have a natural morphism of Artin stacks $\mathcal{A}_{g} \rightarrow\left[E_{\mathcal{Z}} \backslash \mathrm{Sp}_{2 g} \otimes \mathbb{F}_{p}\right]$. It has the property that it maps $\check{\mathcal{R}}_{g}$ onto $\mathcal{R}_{g}$. Our main observation now becomes:

Theorem 1.1. The morphism $\mathcal{A}_{g} \otimes \mathbb{F}_{p} \rightarrow\left[E_{\mathcal{Z}} \backslash \mathrm{Sp}_{2 g} \otimes \mathbb{F}_{p}\right]$ naturally extends to the minimal compactification: $\mathcal{A}_{g}^{*} \otimes \mathbb{F}_{p} \rightarrow\left[E_{\mathcal{Z}} \backslash \mathrm{Sp}_{2 g} \otimes \mathbb{F}_{p}\right]$ and the induced ring homomorphism $\check{\mathcal{R}}_{g} \rightarrow A_{\mathbb{Q}}^{\bullet}\left(\mathcal{A}_{g}^{*} \otimes \mathbb{F}_{p}\right)$ is an embedding.

Here the ring $A_{\mathbb{Q}}^{\bullet}\left(\mathcal{A}_{g}^{*} \otimes \mathbb{F}_{p}\right)$ is Fulton's bivariant Chow ring [9]. One may be tempted to call this image the tautological ring of $\mathcal{A}_{g}^{*}$, although (as was shown in [3]), the stable cohomology of the Baily-Borel compactification is larger than the algebra generated by the $\lambda_{i}$. 
Remark 1.2. A natural analogue of this theorem can be stated for Shimura varieties $X$ of Hodge type, where the role of $\mathcal{R}_{g}$ is taken by the subalgebra $\mathcal{R}_{G} \subset A_{\mathbb{Q}}^{\bullet}(X \otimes k)$, with $k$ a finite field, generated by Chern classes of automorphic bundles. Here the subscript $G$ refers to the algebraic group that is part of the data that give $X$ the structure of a Shimura variety. It is here implicit that we employ an integral model for $X$ which has good reduction over the prime with residue field $k$. Such models have been constructed by Vasiu [25] and Kisin [14].

The work of Pink, Wedhorn and Ziegler [21, 22] applies to this setting: we still have a moduli stack of zips $\left[E_{\mathcal{Z}} \backslash G\right]$ and a classifying morphism $\zeta: X \rightarrow\left[E_{\mathcal{Z}} \backslash G\right]$, the fibres of which are the Ekedahl-Oort strata. The Chow $\mathbb{Q}$-algebra of $\left[E_{\mathcal{Z}} \backslash G\right]$ (here denoted $\check{\mathcal{R}}_{G}$ ) is according to [2, Thm. 2.4.4] isomorphic to that of the compact dual $\check{D}$. If $\zeta$ is faithfully flat and surjective and can be extended to a morphism $\tilde{\zeta}$ of a toroidal compactification of Faltings-Chai type, then essentially the same proof shows that $\check{\mathcal{R}}_{G}$ embeds in the Chow algebra of the toroidal compactification (see [26, 28, 19, 15] for results in this direction). The strata extend to the boundary and enjoy good intersection properties with the boundary, see [1, Thm. 6.1.6] and [16]. The morphism $\tilde{\zeta}$ factors through a morphism $\eta$ of the minimal compactification to the stack $\left[E_{\mathcal{Z}} \backslash G\right]$ and we thus find in a way similar to the case of $\mathcal{A}_{g}$ a copy of $\check{\mathcal{R}}_{G}$ in the Chow algebra of the minimal compactification $A_{\mathbb{Q}}^{\bullet}\left(D_{\Gamma}^{*}\right)$. We will confine ourselves however to the case $\mathcal{A}_{g}$.

Let us note that Esnault and Harris [6] recently proved a lifting property in the case of mixed characteristic, but on the level of $\ell$-adic cohomology. It would be interesting to see whether their result can be lifted to the level of Chow algebras.

Recent work of Wedhorn-Ziegler [27] and Goldring-Koskivirta [11], [12] points towards a possible generalization to Shimura varieties of Hodge type.

\section{The CASE $\mathcal{A}_{g}$}

2.1. Review of the situation in characteristic zero. We let $\tilde{\mathcal{A}}_{g}$ be a toroidal compactification of $\mathcal{A}_{g}$ of Faltings-Chai type and denote by $q$ : $\tilde{\mathcal{A}}_{g} \rightarrow \mathcal{A}_{g}^{*}$ the natural projection. The Hodge bundle $\mathbb{E}^{g}$ on $\mathcal{A}_{g}$ extends to $\tilde{\mathcal{A}}_{g}$ and this extension is again denoted by $\mathbb{E}^{g}$.

The analytic space of the complex fibre $\mathcal{A}_{g}^{\text {an }}$ can be described in terms of the Chevalley group $G=\mathrm{Sp}_{2 g}$, the automorphism group of the standard symplectic lattice $\mathbb{Z}^{2 g}$ as $G(\mathbb{Z}) \backslash D_{g}$ where $D_{g}=G(\mathbb{R}) / K$ is a bounded symmetric domain with $K$ a maximal compact subgroup.

Let us briefly review what is known about the Chow ring of the compact dual of $D_{g}$ in the more general case where $G$ is a reductive algebraic $\mathbb{R}$ group whose symmetric space $D$ has the structure of a bounded symmetric domain. Then the compact dual $\check{D}$ of $D$ is of the form $(G / P)(\mathbb{C})$ with $P$ a maximal parabolic subgroup of $G$. We have a decomposition $G / P$ into 
Schubert cells: $G / P=\bigsqcup B w P / P$, where $w$ runs over the elements of the Weyl group $W$ of $G$, or rather (in order to keep the union disjoint), over a complete set $W^{P}$ of coset representatives for $W / W_{P}$, where $W_{P}$ is the subgroup of $W$ associated to $P$. It is known that the Chow ring $A^{\bullet}(\check{D})$ has as an additive basis the classes of the closures of Schubert cells (Schubert varieties) in $\check{D}$. The ring structure on the Chow ring with $\mathbb{Q}$-coefficients, $A_{\mathbb{Q}}^{\bullet}(\check{D})$, is described by Borel (see $[24$, p. $142,(28)]$ :

$$
A_{\mathbb{Q}}^{\bullet}(\check{D}) \cong \mathcal{S}^{W_{P}} /\left\langle\mathcal{S}_{+}^{W}\right\rangle \text {. }
$$

Here $\mathcal{S}$ is the symmetric $\mathbb{Q}$-algebra on the character group of a Borel subgroup, $\mathcal{S}^{W_{P}}$ is the invariant part under $W_{P}$ and $\left\langle\mathcal{S}_{+}^{W}\right\rangle$ is the ideal generated by $W$-invariant elements of positive degree. In case the group is 'special', e.g. for $\mathrm{GL}_{n}$ and $\mathrm{Sp}_{2 n}$, this isomorphism also holds for $\mathbb{Z}$-coefficients.

In our case, where $G=\mathrm{Sp}_{2 g}$, this graded $\mathbb{Q}$-algebra is isomorphic to

$$
\check{\mathcal{R}}_{g}=\mathbb{Q}\left[u_{1}, \ldots, \ldots, u_{g}\right] / I,
$$

where $u_{i}$ has degree $i$ and $I$ is the ideal generated by the graded pieces of

$$
\left(1+u_{1}+\cdots+u_{g}\right)\left(1-u_{1}+u_{2}-\cdots+(-1)^{g} u_{g}\right)-1 .
$$

So this gives a relation in every positive even degree $\leq 2 g$. Note that $\operatorname{dim}_{\mathbb{Q}} \check{\mathcal{R}}_{g}=2^{g}$.

For a field $k$, the Chern classes $\lambda_{i}:=c_{i}\left(\mathbb{E}^{g}\right)$ in $A_{\mathbb{Q}}^{i}\left(\tilde{\mathcal{A}}_{g} \otimes k\right)$ satisfy the same relation as the $u_{i}$ in the Chow ring of $\tilde{\mathcal{A}}_{g}$ as the $u_{i}$ :

$$
\left(1+\lambda_{1}+\cdots+\lambda_{g}\right)\left(1-\lambda_{1}+\cdots+(-1)^{g} \lambda_{g}\right)=1
$$

(see $[10,7])$ and they generate a subring of the Chow $\operatorname{ring} A_{\mathbb{Q}}^{\bullet}\left(\tilde{\mathcal{A}}_{g} \otimes k\right)$ isomorphic to the rational Chow ring of $\check{D}_{g}$. This extends the Hirzebruch-Mumford Proportionality to the Chow rings. This ring is called the tautological subring of $\left.A_{\mathbb{Q}}^{\bullet}\left(\tilde{\mathcal{A}}_{g} \otimes k\right)\right)$ and denoted again by $\check{\mathcal{R}}_{g}$. Its image in $A_{\mathbb{Q}}^{\bullet}\left(\mathcal{A}_{g}\right)$ under restriction via $j: \mathcal{A}_{g} \otimes k \hookrightarrow \tilde{\mathcal{A}}_{g} \otimes k$ is $\mathcal{R}_{g}=\check{\mathcal{R}}_{g} /\left(\lambda_{g}\right) \cong \check{\mathcal{R}}_{g-1}$.

2.2. The Artin stack of zips. We now restrict to characteristic $p$ and consider $\mathcal{A}_{g} \otimes \mathbb{F}_{p}$ and $\tilde{\mathcal{A}}_{g} \otimes \mathbb{F}_{p}$. The compact dual of Siegel space (or of any symmetric domain) has no obvious counterpart in positive characteristic. But it turns out that there is a good substitute, viz. the Artin stack of zips, that can take on that role for our purposes. Its origin is the so-called Ekedahl-Oort stratification, introduced in [20]. As we will recall below, it has $2^{g}$ strata, and as was shown in $[10,4]$, each of these has the virtue that the cycle class of its closure lies in the tautological subring. For example, we have the (closed) $p$-rank loci $V_{f}(p$-rank $\leq f$ with $0 \leq f \leq g)$ with cycle classes $\left[V_{f}\right]=(p-1)\left(p^{2}-1\right) \cdots\left(p^{g-f}-1\right) \lambda_{g-f}$. Thus the generators of $\check{\mathcal{R}}_{g}$ are represented by these effective cycles. 
The basic definition. For a principally polarized abelian variety $X$ of dimension $g$ over a perfect field of characteristic $p>0$ the de Rham cohomology space $H_{\mathrm{dR}}^{1}(X)$ comes equipped with a non-degenerate alternating form. The Frobenius operator induces a $p$-linear endomorphism of $H=H_{\mathrm{dR}}^{1}(X)$ whose kernel is its Hodge subspace $H^{0}\left(X, \Omega_{X}^{1}\right)$. Both the kernel and the image of this endomorphism are Lagrangian subspaces $F, F^{\prime}$ of dimension $g$. As we will see below, this structure (consisting of a symplectic vector space $H$ and a Frobenius-linear endomorphism $\varphi$ of $H$ whose kernel and image is a Lagrangian subspace) has only finitely many isomorphism types. Such a structure is called a zip and was studied in [18]. (To make the isomorphism type explicit one usually endows kernel and image with filtrations by taking preimages and images of iterates of $\varphi$ and then extends these to self-dual filtrations on $H$ by adding their symplectic perps. This results in a descending filtration (a refinement of the Hodge filtration) $C^{\bullet}$, and an ascending filtration (a refinement of the conjugate filtration) $D_{\bullet}$, connected by the Cartier operator giving Frobenius-linear identifications $\left(C^{i} / C^{i+1}\right)^{(p)} \cong D_{i} / D_{i-1}$. (The dimensions of the intersections of these filtrations determine the isomorphism type. This will however not matter to us in what follows.)

Moduli space and Schubert varieties. In an evident manner we have defined a moduli space $z(H)$ of all zip structures on $H$; it is the moduli space of triples $\left(L_{1}, L_{2}, \varphi\right)$ with $L_{1}, L_{2}$ Lagrangian subspaces of $H$ and $\varphi:\left(H / L_{1}\right)^{(p)} \stackrel{\sim}{\rightarrow} L_{2}$ an isomorphism. If $F(H)$ is the Grassmannian of Lagrangian subspaces of $H$ and $\mathcal{F}_{F(H)} \rightarrow F(H)$ denotes its universal bundle, then $z(H)$ is an open subset in the total space of the exterior tensor product bundle

$$
\operatorname{Fr}_{p}^{*}\left(H \otimes \mathcal{O}_{F(H)} / \mathcal{F}_{F(H)}\right) \otimes \mathcal{F}_{F(H)}=\operatorname{Fr}_{p}^{*}\left(\mathcal{F}_{F(H)}\right)^{\vee} \otimes \mathcal{F}_{F(H)}
$$

over $F(H) \times F(H)$, where $\operatorname{Fr}_{p}$ is the absolute Frobenius on $F(H)$. The group $G=\operatorname{Sp}(H)$ acts in an evident manner on $z(H)$. We shall call the closure of a $G$-orbit in $z(H)$ a Schubert variety.

There are $2^{g}$ such Schubert varieties. This is based on the observation that the relative position of a pair $\left(F, F^{\prime}\right)$ of Lagrangian subspaces (in other words, the $G$-orbit of such a pair) is given by a double coset of $G$ : if $P$ (resp. $P^{\prime}$ ) is the $G$-stabilizer of $F$ (resp. $F^{\prime}$ ), then the $g \in G$ for which $F=g F^{\prime}$ make up the double coset $P g P^{\prime}$, so that we get an element of $P \backslash G / P^{\prime}$. We can identify this set of double cosets in terms of Weyl groups: if we choose a Borel subgroup $B$ contained in $P$ with maximal torus $T$ and $N_{G}(T)$ (resp. $N_{P}(T)$ ) is the normalizer of $T$ in $G$ (resp. in $P$ ), then $W=N_{G}(T) / T$ (resp. is $\left.W_{P}:=N_{P}(T) / T\right)$ is the Weyl group of the pair $(G, T)(\operatorname{resp} .(P, T))$ and it is a standard fact of the theory of algebraic groups that the natural map

$$
W_{P} \backslash W \cong N_{T}(P) \backslash N_{T}(G) \rightarrow P \backslash G / P^{\prime}
$$

is a bijection. One finds that in our case $W_{P} \backslash W$ has $2^{g}$ elements, and hence there are as many Schubert varieties. 
The Artin stack of zip data. Let us make here the connection with the way this notion appears in the literature. The groups $P$ and $P^{\prime}$ are maximal parabolic subgroups of $G$ whose Levi quotients $L_{P}$ resp. $L_{P^{\prime}}$ can be identified with the general linear groups of $F$ (or of its dual $H / F$ for that matter) resp. $F^{\prime}$. So an isomorphism $L_{P} \cong L_{P^{\prime}}^{\prime}$ can be understood as giving an isomorphism $H / F \cong F^{\prime}$ up to a scalar. Similarly, a Frobenius-linear map of $H / F$ onto $F^{\prime}$ determines a Frobenius isogeny $L_{P} \rightarrow L_{P^{\prime}}$. We can formulate this in terms of $G$ only: in our setting a zip datum is given by a 4 -tuple $\mathcal{Z}=\left(G, P, P^{\prime}, \varphi\right)$, where $G=\mathrm{Sp}_{2 g} / \mathbb{F}_{p}, P$ and $P^{\prime}$ are maximal parabolic subgroups of $G$ and $\varphi: L_{P} \rightarrow L_{P^{\prime}}$ is an isogeny between their Levi quotients given by Frobenius. We form the fibre product of $P$ and $P^{\prime}$ over $L_{P^{\prime}}$ (the former via the group homomorphism $P \rightarrow L_{P} \stackrel{\varphi}{\rightarrow} L_{P^{\prime}}$ ) in the category of algebraic groups:

$$
E_{\mathcal{Z}}:=P \times_{L_{P^{\prime}}} P^{\prime}
$$

This group acts on $G$ by $(p, q) \in E_{\mathcal{Z}}: g \mapsto p g q^{-1}$ and we can form the Artin stack $\left[E_{\mathcal{Z}} \backslash G\right]$. Brokemper determined the Chow ring of the stack $\left[E_{\mathcal{Z}} \backslash G\right]$ (which is essentially by definition the $G$-equivariant Chow ring of $z(H)$ ). He considers in [2] more generally the case of a connected group $G$ and an algebraic zip datum. Choose $g \in G$ such that $T^{\prime}:=g T g^{-1} \subset P^{\prime}$. If we identify $T$ resp. $T^{\prime}$ with their images in $L_{P}$ resp. $L_{P^{\prime}}$, then we can even arrange that $\varphi$ takes $T$ to $T^{\prime}$, so that we have defined an isogeny

$$
\widetilde{\varphi}: T \rightarrow T, \quad t \mapsto g^{-1} \varphi(t) g
$$

Then $\widetilde{\varphi}$ acts on $\mathcal{S}$, the symmetric algebra of the character group of $T$. The Chow ring of the stack is ([2, Thm. 2.4.4, page 27])

$$
A^{\bullet}\left(\left[E_{\mathcal{Z}} \backslash G\right]\right)=\mathcal{S}^{W_{P}} /\left(f-\widetilde{\varphi}(f): f \in \mathcal{S}_{+}^{W}\right)
$$

In our case, this group is additively generated by the Schubert varieties as defined above.

This Chow ring can be regarded as the ring of characteristic classes for symplectic vector bundles over $\mathbb{F}_{p}$ endowed with a zip structure for the following reason. If we have a symplectic vector bundle $\mathcal{H}$ over a base scheme $S$ (or stack, for that matter) over $\mathbb{F}_{p}$ of rank $2 g$, then the above construction yields the zip bundle $\mathcal{Z}(\mathcal{H})$ over $S$, so that to endow $\mathcal{H}$ with a zip structure amounts to giving a section of $\mathcal{Z}(\mathcal{H}) / S$. This comes with relative Schubert varieties and these define an embedding of $A^{\bullet}\left(\left[E_{\mathcal{Z}} \backslash G\right]\right)$ in Fulton's bivariant Chow ring $A^{\bullet}(\mathcal{Z}(\mathcal{H}))$ as a subalgebra, having these relative Schubert varieties as additive generators. If a zip structure on $\mathcal{H}$ has associated section $\sigma$, then we may define its ring of characteristic classes as the image of this subalgebra under $\sigma^{*}: A^{\bullet}(Z(\mathcal{H})) \rightarrow A^{\bullet}(S)$. Note that when $\sigma$ has proper intersection with a given relative Schubert variety $Z$ in $Z(\mathcal{H})$, then the associated class $\sigma^{*}[Z]$ is represented by a specific algebraic cycle $\geq 0$ on $S$ defined over $\mathbb{F}_{p}$; we shall refer to these as the Ekedahl-Oort cycles. 
2.3. Degenerations of zips. Let us for a moment return to our fixed symplectic vector space $H$ over $\mathbb{F}_{p}$ and suppose we are given an isotropic subspace $I \subset H$ over $\mathbb{F}_{p}$. Then $H^{\prime}:=I^{\perp} / I$ is a symplectic vector space over $\mathbb{F}_{p}$ and we if assign to a Lagrangian subspace $F \subset H$ which contains $I$ the subspace $F / I \subset H^{\prime}$, we get a bijection between the Lagrangian subspaces of $H$ containing $I$ and the Lagrangian subspaces of $H^{\prime}$. Denote by $z(H, I) \subset z(H)$ the subscheme defined by the Frobenius-linear endomorphisms $\varphi$ of $H$ that are zero on $I$, preserve $I^{\perp}$, and induce the Frobenius on $H / I^{\perp}$. The kernel of $\varphi$ is sandwiched between $I$ and $I^{\perp}$ and the induced endomorphism $\varphi^{\prime}$ of $H^{\prime}$ defines an element of $z\left(H^{\prime}\right)$, as both its kernel and image are Lagrangian subspaces. The resulting morphism $z(H, I) \rightarrow z\left(H^{\prime}\right)$ is equivariant over the evident group homomorphism from the $\operatorname{Sp}(H)$-stabilizer of $I$ to $\operatorname{Sp}\left(H^{\prime}\right)$ and this makes $z(H, I)$ a torsor over a vector bundle on $z\left(H^{\prime}\right)$. The preimage of a Schubert subvariety of $z\left(H^{\prime}\right)$ is contained in a Schubert subvariety of $z(H)$ of the same codimension. To be precise, every $\operatorname{Sp}(H)$ orbit in $z(H)$ orbit meets $z(H, I)$ transversally, and when this intersection is nonempty, then it is the preimage of a $\operatorname{Sp}\left(H^{\prime}\right)$-orbit in $z\left(H^{\prime}\right)$. Recall that the Schubert cells correspond bijectively to the elements of $W_{P} \backslash W$ with $P$ the stabilizer of a Lagrangian $F \subset H$ and similarly the Schubert cells of $z\left(H^{\prime}\right)$ correspond to $W_{P^{\prime}} \backslash W^{\prime}$ with $P^{\prime}$ the stabilizer of $F / I$ and $W^{\prime}$ the Weyl group of $\operatorname{Sp}\left(H^{\prime}\right)$. The map $z(H, I) \rightarrow z\left(H^{\prime}\right)$ is a stratified map corresponding to an embedding $\iota_{I}: W_{P^{\prime}} \backslash W^{\prime} \hookrightarrow W_{P} \backslash W$.

We use these observations to understand a class of degenerations of zips over a discrete valuation ring. Let $R$ be a discrete valuation ring of finite type over $\mathbb{F}_{p}$ with residue field $\kappa$ and field of fractions $K$.

Let $\mathcal{H}$ denote a symplectic space of rank $2 g$ over $R$ and $\mathcal{I} \subset \mathcal{H}$ an isotropic subspace over $R$ (so that $\mathcal{H}^{\prime}:=\mathcal{I}^{\perp} / \mathcal{I}$ is a symplectic space over $R$ ). If $H:=\kappa \otimes_{R} \mathcal{H}$ with isotropic subspace $I$ (resp. $H^{\prime}:=\kappa \otimes_{R} \mathcal{H}^{\prime}$ ) denotes the fiber over the closed point, then we have evident specialization maps $z(\mathcal{H}) \rightarrow z(H)\left(\operatorname{resp} . z\left(\mathcal{H}^{\prime}\right) \rightarrow z\left(H^{\prime}\right)\right)$

Suppose given $\varphi \in \mathcal{Z}(\mathcal{H}, \mathcal{I})$ and assume that $\varphi_{K}$ belongs to the Schubert cell with index $w$. We let $\varphi^{\prime}$ be the image in $\mathcal{Z}\left(\mathcal{H}^{\prime}\right)$ with specialization $\varphi_{o}^{\prime} \in \mathcal{Z}\left(H^{\prime}\right)$. The discussion above implies the following result.

Lemma 2.1. If the element $\varphi_{K}$ belongs to the Schubert cell with index $w$ and $\varphi_{o}^{\prime}$ to the Schubert cell $w^{\prime}$, then the specialization $\varphi_{o}$ belongs to the Schubert cell with index $\iota_{I}\left(w^{\prime}\right)=w$.

2.4. Extension of the stratification across the Satake compactifica-

tion. By assigning to a principally polarized abelian variety of dimension $g$ the isomorphism type of its zip on its first de Rham cohomology space, we obtain a stratification of the moduli space $\mathcal{A}_{g} \otimes \mathbb{F}_{p}$, the Ekedahl-Oort stratification. It is is induced by a morphism of stacks

$$
\zeta: \mathcal{A}_{g} \rightarrow\left[E_{\mathcal{Z}} \backslash G\right]
$$

This morphism is smooth (see [28, Thm. 4.1.2]) and the fibres are the strata. 
This stratification can be extended to a toroidal compactification (of ChaiFaltings type) $\tilde{\mathcal{A}}_{g} \otimes \mathbb{F}_{p}$. The space $\tilde{\mathcal{A}}_{g}$ admits a stratification by torus rank: if $q: \tilde{\mathcal{A}}_{g} \rightarrow \mathcal{A}_{g}^{*}$ is the canonical map to the Baily-Borel compactification and $\mathcal{A}_{g}^{*}=\sqcup_{i=0}^{g} \mathcal{A}_{g-i}$ is the standard decomposition, then the restriction of the Hodge bundle to $\mathcal{A}_{g}^{\langle g-i\rangle}:=q^{-1}\left(\mathcal{A}_{g-i}\right)$, contains a rank $g-i$ subbundle $\mathbb{E}^{(g-i)}$ which is the pullback of the Hodge bundle on $\mathcal{A}_{g-i}$.

The canonical extension of the de Rham complex is the logarithmic de Rham complex where logarithmic singularities are allowed along the divisor added to compactify the semi-abelian variety, cf. [8, VI, Theorem 1.1, p. 195]. The logarithmic de Rham sheaf

$$
\mathcal{H}^{1}:=R^{1} \pi_{*}\left(\Omega_{\tilde{\mathcal{X}}_{g} / \tilde{\mathcal{A}}_{g}}(\log )\right)
$$

extends the de Rham sheaf $\mathcal{H}_{\mathrm{dR}}^{1}\left(\mathcal{X} / \mathcal{A}_{g}\right)$. On $\tilde{\mathcal{A}}_{g} \otimes \mathbb{F}_{p}$ it comes again with two filtrations forming a zip. In fact, the morphism $\zeta$ can be extended to a morphism $\tilde{\zeta}: \tilde{\mathcal{A}}_{g} \otimes \mathbb{F}_{p} \rightarrow\left[E_{\mathcal{Z}} \backslash G\right]$ which is again smooth as can be seen by using [4, Lemma 5.1] or [1], see also below. The closed strata on $\tilde{A}_{g} \otimes \mathbb{F}_{p}$ are the closures of the strata on $\mathcal{A}_{g} \otimes \mathbb{F}_{p}$.

The Ekedahl-Oort stratification on $\tilde{\mathcal{A}}_{g} \otimes \mathbb{F}_{p}$ intersects the boundary strata transversally as we will now explain. The reason is that the Ekedahl-Oort stratification is defined by the action of Frobenius and Verschiebung acting on the logarithmic de Rham cohomology $H_{\mathrm{dR}}^{1}$ of a semi-abelian variety and on the toric part this action is essentially trivial.

We consider a semi-abelian variety $\mathcal{G}^{0}$ over $S=\operatorname{Spec}(R)$ with $R$ a discrete valuation ring of finite type over $\mathbb{F}_{p}$. We assume that the generic fibre is abelian and the special fibre is the Néron model of a semi-abelian variety of torus rank $r$. We let $\mathcal{G} / S$ be a toroidal compactification of $\mathcal{G}^{0}$ of FaltingsChai type. It can be obtained via the action on a semi-abelian variety $\tilde{\mathcal{G}}$ over $S$ by a group of periods $\iota: Y \rightarrow \tilde{\mathcal{G}}(S)$ with $Y$ free abelian of rank $r$. Here the semi-abelian variety $\tilde{\mathcal{G}}$ is an extension $0 \rightarrow T \rightarrow \tilde{\mathcal{G}} \rightarrow A \rightarrow 0$ of an abelian scheme $A / S$ by a split torus $T / S$ of rank $r$. In this case the logarithmic de Rham cohomology can be described with the help of universal vector extensions, that is, extensions of group schemes by vector group schemes. We refer to [8] pages 81-86 for a description. The universal vector extension $E_{\tilde{\mathcal{G}}}$ of $\tilde{\mathcal{G}}$ is a vector group extension

$$
0 \rightarrow L_{\tilde{\mathcal{G}}} \rightarrow E_{\tilde{\mathcal{G}}} \rightarrow \tilde{\mathcal{G}} \rightarrow 0
$$

that is canonically isomorphic to the pullback under $\tilde{\mathcal{G}} \rightarrow A$ of the universal vector extension $0 \rightarrow L_{A} \rightarrow E_{A} \rightarrow A \rightarrow 0$ of $A$, where $L_{A}=\operatorname{Lie}\left(A^{\vee} / S\right)^{\vee}$ is the sheaf of invariant relative 1 -forms on the dual abelian variety $A^{\vee}$ of $A$. For the quotient construction we need an equivariant form of this, that is, we need in addition a lifting of the homomorphism $Y \rightarrow \tilde{\mathcal{G}}(S)$ to $Y \rightarrow E_{\tilde{\mathcal{G}}}(S)$. Then $Y$ acts via translation. 
The dual of the logarithmic de Rham cohomology $\mathcal{H}^{1}$ is the $Y$-equivariant Lie-algebra of the universal vector extension of $\tilde{\mathcal{G}}$. By the toroidal construction as in $[8, \mathrm{Ch}$. VI] this Lie-algebra has a weight filtration with subquotients the Lie algebra $L_{T}$ of $T$, the homology of the abelian variety $A$ and $Y$. The ranks are $r, 2 g-2 r$ and $r$. The subspace of rank $2 g-r$ of $\mathcal{H}^{1}$ will be denoted by $I^{\perp}$ and its orthogonal complement by $I$. We can identify $I$ with the invariant differentials of the torus $T$. Then $I$ is isotropic and contained in the kernel of Frobenius. We are thus in the situation described above in subsection 2.3. Since $I$ is contained in the kernel of Frobenius the isomorphism type of the zip on the special fibre of $\mathcal{H}^{1}$ depends only on the zip of the de Rham cohomology of the abelian part. We can apply Lemma 2.1 to conclude that the closures of the strata on $\mathcal{A}_{g} \otimes \mathbb{F}_{p}$ are the strata on $\tilde{\mathcal{A}}_{g} \otimes \mathbb{F}_{p}$ and by induction that the intersection with the boundary strata is proper. Indeed, with the notation used there, if $\varphi_{K} \in \mathcal{Z}\left(\mathcal{H}_{K}, \mathcal{I}_{K}\right)$ and $\varphi_{K}^{\prime}$ belongs to the Schubert cycle with index $w^{\prime} \in W_{P^{\prime}} \backslash W^{\prime}$ then $\varphi_{K}$ extends uniquely to $\varphi$ with Schubert index $\iota_{I}\left(w^{\prime}\right)$.

Remark 2.2. The valuation of the torus part of the periods defines a $\mathbb{Z}$ valued bilinear form on $Y$ which we can see as the analogue of the monodromy operator of Hodge theory. Its invariant part defines a subspace $I^{\perp}$ of dimension $2 g-r$ in the special fibre of the logarithmic de Rham cohomology over $S$. (One might view it as associated to the Dieudonné module of the kernel of multiplication by $p$ on the semi-abelian special fibre of $\mathcal{G}$.)

We thus see that the map $\tilde{\zeta}: \tilde{\mathcal{A}}_{g} \otimes \mathbb{F}_{p} \rightarrow\left[E_{\mathcal{Z}} \backslash G\right]$ factors through a map

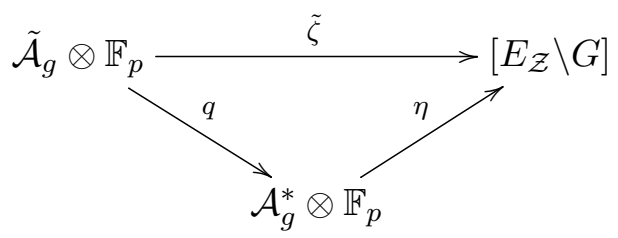

The morphism $\tilde{\zeta}: \tilde{\mathcal{A}}_{g} \otimes \mathbb{F}_{p} \rightarrow\left[E_{\mathcal{Z}} \backslash G\right]$ induces a homomorphism of Chow rings

$$
A_{\mathbb{Q}}^{\bullet}\left(\left[E_{\mathcal{Z}} \backslash G\right]\right) \rightarrow A_{\mathbb{Q}}^{\bullet}\left(\tilde{\mathcal{A}}_{g} \otimes \mathbb{F}_{p}\right)
$$

and it induces an isomorphism $A_{\mathbb{Q}}^{\bullet}\left(\left[E_{\mathcal{Z}} \backslash G\right]\right) \cong \check{\mathcal{R}}_{g}$. Indeed, the closed Ekedahl-Oort strata on $\tilde{A}_{g} \otimes \mathbb{F}_{p}$ are effective cycles with non-zero classes.

Proof of Theorem 1.1. The image under push forward via $q: \tilde{\mathcal{A}}_{g} \rightarrow \mathcal{A}_{g}^{*}$ of $\lambda_{i} \in A_{\mathbb{Q}}^{i}\left(\tilde{\mathcal{A}}_{g}\right)$ in the Chow cohomology group $A_{\mathbb{Q}}^{i}\left(\mathcal{A}_{g}^{*}\right)$ is independent of the chosen toroidal compactification, see [5, Def-Prop. 3.1]. Thus these define classes $\lambda_{i}^{\prime}$ in $A_{\mathbb{Q}}^{i}\left(\mathcal{A}_{g}^{*}\right)$. On the other hand we have the generators $\underline{\lambda}_{i}$ of the Chow ring of the stack $\left[E_{\mathcal{Z}} \backslash G\right]$ and via the map $\eta: \mathcal{A}_{g}^{*} \otimes \mathbb{F}_{p} \rightarrow\left[E_{\mathcal{Z}} \backslash G\right]$ these act as bivariant classes by cap product $\cap \underline{\lambda}_{i}: A_{k}\left(\mathcal{A}_{g}^{*} \otimes \mathbb{F}_{p}\right) \rightarrow A_{k-i}\left(\mathcal{A}_{g}^{*} \otimes \mathbb{F}_{p}\right)$ 
on the Chow groups of $\mathcal{A}_{g}^{*} \otimes \mathbb{F}_{p}$. These satisfy $\tilde{\zeta}^{*}\left(\underline{\lambda}_{i}\right)=\lambda_{i}$. By $[9,17.1]$ and the projection formula $([9$, p. 323]) we have

$$
\cap \underline{\lambda}_{i}\left(q_{*}(c)\right)=q_{*}\left(\lambda_{i} \cdot c\right)=\lambda_{i}^{\prime} q_{*}(c)
$$

for all $c \in A_{k}\left(\tilde{\mathcal{A}}_{g}\right)$. This enables us identify the bivariant classes $\underline{\lambda}_{i}$ with the $\lambda_{i}^{\prime}$. It thus gives rise to a diagram

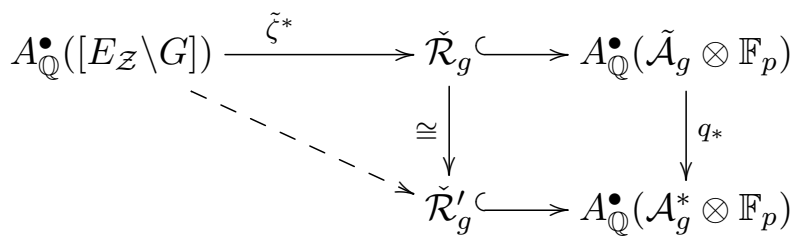

Remark 2.3. In the end the argument is based on the observation that all the tautological classes $\lambda_{i}$ have an effective representative on $\tilde{\mathcal{A}}_{g} \otimes \mathbb{F}_{p}$ that intersects the boundary properly. This fails to be so in characteristic zero, although it is then true for the ample $\lambda_{1}$, and hence for any power on $\lambda_{1}$, like $\lambda_{2}=(1 / 2) \lambda_{1}^{2}$. But this is not so for $\lambda_{3}$. This seems related to the question of whether for a given field $k$ the space $\mathcal{A}_{g} \otimes k$ contains complete subvarieties of codimension $g$. For $g=3$ every complete subvariety of $\mathcal{A}_{3} \otimes k$ has as class a multiple of $\lambda_{3}$. Conversely, an effective representative for $\lambda_{g}$ transversal to the boundary of $\tilde{\mathcal{A}}_{g} \otimes k$ does not intersect the boundary because $\lambda_{g}^{2}=0$, hence yields a complete subvariety of codimension $g$.

\section{ACKNOWLEDGEMENTS}

We thank Luc Illusie and Gerd Faltings for correspondence and the referee for useful suggestions.

\section{REFERENCES}

[1] G. Boxer: Torsion in the coherent cohomology of Shimura varieties and Galois representations, Ph.D. thesis, Harvard University, Cambridge, Massachusetts, USA, 2015. 3, 8

[2] D. Brokemper: On the Chow ring of the stack of truncated Barsotti-Tate groups, Pacific J. Math. 296 (2018), 271-303. arXiv:1611.08900v1. 3, 6

[3] R. Charney, R. Lee: Cohomology of the Satake compactification, Topology 22 (1983), 389-423. 2

[4] T. Ekedahl, G. van der Geer: Cycle classes of the E-O stratification on the moduli of abelian varieties, in: Algebra, Arithmetic, and Geometry, Y. Tschinkel and Y. Zarhin (eds.), Progress in Mathematics 269, Birkhäuser, Basel (2010). 2, 4, 8

[5] T. Ekedahl, G. van der Geer: Cycles representing the top Chern class of the Hodge bundle on the moduli space of abelian varieties. Duke Math. Journal 129 (2004), 187-199. 9

[6] H. Esnault, M. Harris: Chern classes of automorphic vector bundles, II, Épijournal Geom. Algébrique 3 (2019), Art. 14, 28 pp. https://arxiv.org/pdf/1801.08219. pdf 3

[7] H. Esnault, E. Viehweg: Chern classes of Gauss-Manin bundles of weight 1 vanish. K-Theory 26 (2002), 287-305. 4 
[8] G. Faltings, C.L. Chai: Degeneration of abelian varieties. Ergebnisse der Math. 22, Springer-Verlag, 1990. 8, 9

[9] W. Fulton: Intersection Theory. Ergebnisse der Mathematik und ihrer Grenzgebiete. 2 1984. Springer Verlag. 2, 10

[10] G. van der Geer: Cycles on the moduli space of abelian varieties, in: Moduli of curves and abelian varieties, Aspects Math., E33, Vieweg, Braunschweig (1999) 4

[11] W. Goldring, J.-S. Koskivirta: Strata Hasse invariants, Hecke algebras and Galois representations. Invent. Math. 217 (2019), 887-984. https://arxiv.org/pdf/ 1507.05032.pdf 3

[12] W. Goldring, J-S. Koskivirta: Stratification of flag spaces and functoriality. Int. Math. Res. Not. 2019 (2019), 3646-3682. https : / /arxiv .org/pdf/1608.01504v2. pdf. 3

[13] M. Goresky, W. Pardon: Chern classes of automorphic vector bundles, Invent. Math. 147 (2002), 561-612. 2

[14] M. Kisin: Integral models for Shimura varieties of abelian type. J. Amer. Math. Soc. 23 (2010), 967-1012. 3

[15] M. Kisin: Honda-Tate theory for Shimura varieties. Oberwolfach Report No. 39/2015, "Reductions of Shimura varieties". 3

[16] K.-W. Lan, B. Stroh: Compactifications of subschemes of integral models of Shimura varieties. Forum of Mathematics, Sigma (2018), 6, 105 pages 3

[17] E. Looijenga: Goresky-Pardon lifts of Chern classes and associated Tate extensions, Compositio Math. 153 (2017), 1349-1371. 2

[18] B. Moonen, T. Wedhorn: Discrete invariants of varieties in positive characteristic. Int. Math. Res. Not., 72: 3855-3903, 2004. 2, 5

[19] S. Nie: Fundamental elements of an affine Weyl group. Math. Ann. 362 (2015), 485-499. 3

[20] F. Oort: A stratification of a moduli space of polarized abelian varieties in positive characteristic, in: Moduli of Abelian Varieties, Progr. Math. 195, Birkhäuser, 2001, pp. 345-416. 4

[21] R. Pink, T. Wedhorn, P. Ziegler: Algebraic zip data. Documenta Math. 16 (2011), pp. 253-300. 2, 3

[22] R. Pink, T. Wedhorn, P. Ziegler: F-zips with additional structure. Pacific J. Math. 274 (2015), 183-236. 3

[23] T. Springer: An extension of Bruhat's lemma. Journal of Algebra 313 (2007), $417-427$

[24] H. Tamvakis: Giambelli degeneracy locus formulas for classical $G / P$ spaces. Moscow Math. Journal 16 (2016), 125-177. 4

[25] A. Vasiu: Integral canonical models of Shimura varieties of preabelian type, Asian J. Math. 3 (1999), 401-518. 3

[26] E. Viehmann and T. Wedhorn: Ekedahl-Oort and Newton strata for Shimura varieties of PEL type. Math. Ann. 356 (2013), 1493-1550. 3

[27] T. Wedhorn, P. Ziegler: Tautological rings of Shimura varieties and cycle classes of Ekedahl-Oort strata. https://arxiv.org/pdf/1811.04843.pdf 3

[28] C. Zhang: Ekedahl-Oort strata for good reductions of Shimura varieties of Hodge type. Canad. J. Math. 70 (2018), 451-480. https://arxiv.org/pdf/1312.4869. pdf. 3,7 
Korteweg-de Vries Instituut, Universiteit van Amsterdam, Amsterdam, The Netherlands and Yau Mathematical Sciences Center, Tsinghua University BEIJING, CHINA.

E-mail address: g.b.m.vandergeer@uva.nl

Yau Mathematical Sciences Center, Tsinghua University Beijing (China) and Mathematisch Institudt, Universiteit Utrecht (Nederland)

E-mail address: e.j.n.looijenga@uu.nl 